\title{
RECOVERY OF DISASTER IN CLOUD ENVIRONMENT - STUDY
}

\author{
Lalitha M \\ Department of Computer Science \\ Tamil Nadu Open University \\ Chennai, Tamil Nadu, India
}

\author{
Dr. R Kalaiarasi \\ Department of Computer Science \\ Tamil Nadu Open University \\ Chennai, Tamil Nadu, India
}

\begin{abstract}
Data recovery assistance required in today's world for producing information in huge sum. Regardless of systems are down, the cloud service providers provide security to the client. A lot of client's private information is stored in cloud environment. The needs for recovery of data services are growing and an efficient powerful data Rescue methods to be developed in case of information lost In a disaster. The inclination behind recovery strategy is to collect information from alternate server if the server lost information and lacking the ability to provide data to the client. To accomplish this task, many distinct procedures have been proposed. Situations like Fire, Natural calamities or any accidental deletion of data cannot be accessed once more. The scope of this recovery is to focus on enormous data recovery procedures that are used as a part of Cloud computing area. In addition, Identifying open issues and recovery stages are described.
\end{abstract}

\section{Keywords - Data recovery, Recovery of data services}

\section{INTRODUCTION}

Cloud Computing enables organizations to Accumulate and retrieve data over the internet instead of using own computer hardware for distribution of resources. Client has connected with Cloud server and can access information from anyplace through cloud storage. An unexpected interruption can affect Business stability and substantial losses of commercial and finance fame and share. To identify disaster is the problematic scenario in most of the organizations

The disaster leads to enormous loss of data. The disaster can be caused by human beings or natural. Some of the causes of data loss are Floods, Fires, Earthquakes, Power failure, theft. Organization need to safeguard the data from these circumstances when a disaster occurs. Some recovery techniques should be followed to recover the data at the time

Of disaster events.

Traditional method is backing up of disaster data retrieval. The cloud computing provides economical DRPs for small or medium sized business. Many organizations evolve various recovery techniques for business continuity. A disaster recovery process should be documented and maintained by organization. Organization's goal should be fixed plainly, and DRP would be ideal for calamity recuperation.

\section{RELATED WORK}

Several researches have been carried out on cloud disaster recovery and techniques. Few techniques are presented here. Classification of cloud utilized to save the application information after a human interruption or administrator interruption is a unique are by allowing a full data recovery in the cloud. This method is called as catastrophe retrieval service. Data Recovery replication and supporting physical or virtual servers by outsider to overcome failures if there is any man-made or consistent disaster. A new cloud facility model, disaster recovery as a cloud service, proposed by Author Wood for site applications which demonstrate that information backup built on high performance cloud resources can greatly reduce the price of data disaster. Dramas differs from Cloud based benefits by securing data information and encourages more fast application recovery with standby processing limit. Dramas in cloud recovered resources are paid for when they are used, making it more flexible than an ordinary catastrophe restoration site where the recuperation resources must run continuously.

\section{A. Cloud Standby Implementation for Disaster Recovery} In Cloud: [1]'

Utilization of operational standby locales with frequently refreshed standby frameworks is a magnificent way to deal against disasters. The cost of setting and keeping second data center is highly expensive. The strategy of catastrophe retrieval based on deployment model which includes explanation and depiction language.

\section{B. Efficient and Secured Method for Faster Data Restoration \& Availability in Disaster Cloud Data Management: [3]'}

Client registers in CSP to use the services. Depends upon the work File systems are used for registration of three directories. Towards the bulk transfer, the information transferred through the client is encoded in Data transferring stages. For managing disaster issues, the data is formerly backing up to the server and it's encrypted in another protected index. In the Data downloading stage, information can be accessible the demand through the client and information can be accessible on the minute. The second case where rebuilding procedure 


\section{International Journal of Engineering Applied Sciences and Technology, 2019 Vol. 4, Issue 5, ISSN No. 2455-2143, Pages 401-404 \\ Published Online September 2019 in IJEAST (http://www.ijeast.com)}

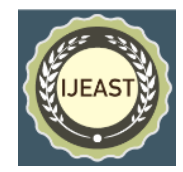

Happens at the time of disaster and client request is sent to an alternate server.

\section{Back up and disaster recovery system for cloud Computing: [2]'}

It is necessary to save information frequently to recover information in case of any disaster. Periodic incremental backup is need in case of low recurrence of changes if the data is generally static.

\section{Discovering Disaster Recovery Parameters in an Enterprise Application: [4]'}

It Deals with unanticipated disturbances that causes vast economic and fame damages to the administrations. This study is based on identifying parameters that impact the catastrophe recovery. These parameters include Controlling and Authorized necessities, Credentials of right set of shareholders.

\section{TRADITIONAL DiSASTER RECOVERY}

\section{A. Tier 0: No offsite data:}

Data is secured in an occurrence of disaster using magnetic tapes, removable disks during backup process.

No offsite data can be defined as the no catastrophe retrieval strategy and no protected information. That means Recovery of documents may take weeks besides can be unsuccessful.

\section{B. Tier 1: Backup with no host site:}

The data is not backed up by hot site but by offsite data transfer methods. The retrieval of data that is stored would take time. No unnecessary servers of their own time taken to progress towards race and organize the administrations. An organization must be set up to acknowledge many days to weeks, yet the reinforcements are secured off-site.

\section{Tier 2: Data backup with hot site:}

It means every organization should preserve data standby servers as well as hot site. We can run applications at standby servers by having a hot reinforcement when disaster occurs. This hot site backup result in data recovery for hours or days to recover information, but the period of recovery can be predicted.

\section{Tier 3: Electronic vaulting:}

This method offers electronic vaulting an alternate for physical backup like tapes. The files are backed up and transmitted to a secured storage location which contains high speed circuit communication. Hot location reinforcement is expensive when compared to get data by net through electronic vaults.

\section{E. Tier 4: Point in time copies:}

It requires extensive information exchange and quicker data recovery than clients of inferiorities. PIT means uses and preserves backup of critical data which is accessible to backup site using web.

\section{F. Tier 5: Transaction integrity:}

This solution is useful in consistent data recovery between the data site and the recovery data sites in the business. The integrity and functionality is depends upon the application which is use.

\section{G. Tier 6: Zero or near data loss:}

The data concurrency industries are maintained by BCP which bring back information to tenders in a fast way. To provide data consistency, it doesn't depend on the application. The requirement of disk mirroring and provides synchronous results for the storage retailers. It depends on sort of information exist on tape and amount of data is stored and recovered during disaster.

\section{H. Tier 7: Highly automated, business integrated solution:}

It protects consistency of data that which is agreed by minimal data loss solutions. The recovery or data is automated and allows for restoring of data to systems such that application becomes much faster and reliable. In traditional data redundancy, requires data centers with well- equipped to store data whenever it is backed up. Organization needs several kinds of hardware and software to geo- redundant localities to assure recovery time objective. By organizing the hardware virtualization ease the conventional disaster recovery.

With virtualization, the time of restoration take lesser hours by construction and purpose of critical assistance and identifying parameters for data recovery. The configuration of hardware on recovery spot must be identical to the primary place such that it can carry the entire traffic load by affected site. When the applications are booted from disasters the RTO on virtual machine would be similar to the RTO on customary standby site configuration.

\section{DISASTER RECOVERY REQUIREMENTS}

The main effective cloud disaster recovery key features are RTO and RPO. It helps organization to select ideal backup plan. It contributes basis to diagnose and analyze the procedure in recovery plans.

\section{Recovery Point Objective:}

The period taken to recover the information after a disaster happens is classed as RPO. The requirement of this RPO is that 


\section{International Journal of Engineering Applied Sciences and Technology, 2019 \\ Vol. 4, Issue 5, ISSN No. 2455-2143, Pages 401-404 \\ Published Online September 2019 in IJEAST (http://www.ijeast.com)}

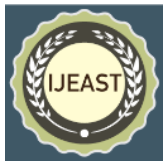

Application data cant' be lost, also helps in continuous synchronous replication. Some application is allowed for acceptable data loss like range sec to hours or a day. The occurrence of catastrophe, the degree of data is lost is recognized. The management of data backup and saving method can be done by RPO. Offline data backups can assist data failure locations within a week of loss of information. Reinforcement of offsite backups should be followed regularly in organization.

Daily on-site backups are required in an organization to encourage the production environment loss with a day of loss of data. In addition, reproducing the transactions at the time of retrieval of data is required after the loss of application. A network area storage assist damage of a site without instances for record correction by no data or information loss. A grouped database helps in the loss of certain storage devices without files loss. Data hub helps in sustain loss of individual data sites with no data loss.

\section{Recovery Time Objective:}

The time duration between disaster and replacement of services includes intrusion detection. At the time of execution, the essential servers are places at backup sites which will prepare the system which is broken. At the time of catastrophe, it recognizes the downtime. To increase disaster recovery performance, synchronous replication of application is used at the time of catastrophe. Disaster recovery should have these effective requirements to reduce RPO and RTO on the regular process. The application should be returned to a regular state and must ensure privacy and confidentiality.

\section{DisASTER RECOVERY Plan}

When disaster recuperation the following five strategies are utilized few components are executed for information reinforcement. The backup places can come from three distinctive sources such as organizations master in providing catastrophe retrieving services, areas operated and maintained by own organization and mutual understanding with other organization to share information in the occurrence of a disaster.

\section{A. Hot Backup Site:}

It is very expensive method for the real time process in organizations. The loss of data is minimal and restores data efficiently.

\section{B. Cool backup site:}

This method doesn't include hardware deployment and backup of data. In this method, all the data must be restored and delivered to the site. It is less expensive than Hot Backup site.

\section{Warm backup site:}

This method includes hardware calibration arranges the secondary or backup location on the primary site. When compared to conventional disaster recuperation DR is cloud is cheaper service which replicates virtually and physically
Flexible. Recovery strategies are inevitable for few working applications. It consists of security, network connectivity, prefabricated selections and server failover. Whenever disaster occurs the data backup can be done using running applications on cloud until we retrieve backup of data to primary site.

The construction of Dramas is explained by three models: From Cloud: when the information in cloud and backup location is in private information center. In cloud: The primary site and backup site are available in cloud. To cloud: The application available in primary data center and recovery location and backup present in cloud. The data is accessed only by organization's administrator. Standard Data recovery solutions are pre-packaged and failover to a cloud can be based on pay-per-use in cloud environment with different rates in Recovery Point Objective (RPO) and Recovery Time Objective (RTO).

\section{Challenges Encounter in Cloud Disaster Recovery:}

- Dependency: This is one of the obstructions of cloud where a client has no procedure or control over data and their system.

- Cost: DR is low at cost. Cloud provides affordable way of mechanism at distinct cost.

- Detection of failure: Failure in detecting the impact result in framework downtime. So it is basic to recognize and report detection at the earliest opportunity for a quick and right Disaster Recovery.

- Security: Disaster can be built by environment or humanmade. Cyber terrorism is one of most human-made failures, can be refined because of some reasons.

- Data Storage: Increase in usage of cloud in market and business it is necessity to store vast quantity of information on cloud established storages. In order to satisfy applications and guarantee the security of data, computing has to distribute nevertheless storage needs to be unified. Therefore storage single point of failure and data loss is critical challenges to store data in cloud.

\section{Maximizing Resource Utilization:}

The utilization of DR services must be increased by CSP's and should guarantee the services at the same time.

Correlated Failures: If a disaster is occurred in an area which leads to high interruption of services, many customers approaches CSP to recover the data. The main task in instance is to allocate clients between servers in such a way that they can reduce correlated failure risk 


\section{International Journal of Engineering Applied Sciences and Technology, 2019 Vol. 4, Issue 5, ISSN No. 2455-2143, Pages 401-404 \\ Published Online September 2019 in IJEAST (http://www.ijeast.com)}

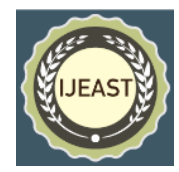

\section{Privacy and confidentiality:}

The data centers of Private firms would be failover through cloud at the time of disaster. Cloud should assure the confidentiality and privacy of data resources which were used for Data Recovery Mechanisms. It also guarantees the application performance that wouldn't get affected by disasters.

\section{Monitoring of Disaster:}

The Qu's Report should be delivered to firms by the failure tolerance. In case of disaster, the faster catastrophe detection in primary site or backup is needed. The main task is how to detect disaster and rank them in initial stages.

Resource Scheduling: The complexities of infrastructure are increasing because of gradual growing of cloud services. Thus, sharing of resources is one of the main problems in the model based cloud environment. The consideration of unpredictable arrival of customers and various disaster situations in cloud Data recovery platforms is important. We require more efficient scheduling techniques for resource scheduling.

\section{CONCLUSION}

The organization must recognize the possible situations where the root disasters and the effect. The goals to be fixed assess efficient DR techniques to pick the Data Recovery Process would be ideal. This paper analyses and displays rules for selecting disaster recovery alternatives. The ideal disaster recovery arranging must observe the main guidelines with the underlying cost, the rate of information exchanges, and the charge of information stockpiling. The requirement of information in an organization and its data recovery should be considered. At the time of risk, the type of disaster can be either normal or human-made must be recognized. The cost effective techniques should be resolved to allow an evaluation of Disaster Recovery Plans related to time for retrieving loss of information (RPO). This will help the organization to control future advancement of the arrangement and maintenance of Data Recovery Plan.

\section{REFERENCE}

[1]

https://www.researchgate.net/publication/326552374_Effi cient_and_Reliable_Data_Recovery_Technique_in_Cloud _Computing

[2] Pro Data Backup and Recovery 1st Ed. Edition - Steven Nelson (Author)

[3] https://searchdisasterrecovery.techtarget.com/definition/d ate-recovery

[4] Data Recovery with \& without Programming Taren Tragi

[5] https://www.semanticscholar.org/paper/A-Survey-onResource-Scheduling-in-Cloud-Computing\%3A-SinghChana/713fbe049deb2bdd01ac07f25ccf4e015b16c98e
[6] Distinguishing cloud computing from utility computing. (http://www.ebizq.net/blogs/saasweak/ 2008/03)

[7] Grumman, Galen (2008-04-07). "What cloud computing really means". InfoWorld. http://www.infoworld.com/d/cloudcomputing/what-cloudcomputing-really-means031. Retrieved 2009-06-02.

[8] "Cloud Computing: Clash of the clouds". The Economist. 2009-10-15.

http://www.economist.com/displaystory.cfm?stor ibid=14637206. Retrieved 2009-11-03.

[9] P. Mell and T. Grace, "Cloud Computing Definition", National Institute of Standards and Technology, Version 15, 10-7-09

[10] L. Wang, G. Laszewski, M. Kunze and J. Tao, "Cloud computing: a perspective study", J New Generation Computing, 2010, pp 1-11.

[11] G. Wei, V. Athanasios, Y. Zheng and N. Xiong, "A game-theoretic method of fair resource allocation for cloud computing services," J. Supercomputing, 2009, DOI 10.1007/s11227-009- 0318-1.

[12] G. Wei, V. Athanasios, Y. Zheng and N. Xing, "A gametheoretic method of fair resource allocation for cloud computing services," J. Supercomputing, 2009, DOI 10.1007/s11227-009- 0318-1.

[13] Cloud Security Alliance. Top Threats to Cloud Computing, 2010. http://www.cloudsecurityalliance.org [accessed on: March, 2010].

[14] Heister J. What you need to know about cloud computing security and compliance, Gartner, Research, and ID Number: G00168345, 2009.

[15] Boss G, Malady P, Quant D, Lagrange L, Hall H. Cloud computing, 2009. http://www.ibm.com/developerswork/websphere /zones/hippos/ library.html. 